\title{
Robust Floor Determination Algorithm for Indoor Wireless Localization Systems under Reference Node Failure
}

\author{
Kriangkrai Maneerat, ${ }^{1}$ Kamol Kaemarungsi, ${ }^{2}$ and Chutima Prommak ${ }^{1}$ \\ ${ }^{1}$ School of Telecommunication Engineering, Suranaree University of Technology, Nakhon Ratchasima, Thailand \\ ${ }^{2}$ National Electronics and Computer Technology Center, NSTDA, Pathumthani, Thailand \\ Correspondence should be addressed to Chutima Prommak; cprommak@sut.ac.th
}

Received 15 July 2016; Accepted 15 September 2016

Academic Editor: Luis J. G. Villalba

Copyright (c) 2016 Kriangkrai Maneerat et al. This is an open access article distributed under the Creative Commons Attribution License, which permits unrestricted use, distribution, and reproduction in any medium, provided the original work is properly cited.

\begin{abstract}
One of the challenging problems for indoor wireless multifloor positioning systems is the presence of reference node (RN) failures, which cause the values of received signal strength (RSS) to be missed during the online positioning phase of the location fingerprinting technique. This leads to performance degradation in terms of floor accuracy, which in turn affects other localization procedures. This paper presents a robust floor determination algorithm called Robust Mean of Sum-RSS (RMoS), which can accurately determine the floor on which mobile objects are located and can work under either the fault-free scenario or the RNfailure scenarios. The proposed fault tolerance floor algorithm is based on the mean of the summation of the strongest RSSs obtained from the IEEE 802.15.4 Wireless Sensor Networks (WSNs) during the online phase. The performance of the proposed algorithm is compared with those of different floor determination algorithms in literature. The experimental results show that the proposed robust floor determination algorithm outperformed the other floor algorithms and can achieve the highest percentage of floor determination accuracy in all scenarios tested. Specifically, the proposed algorithm can achieve greater than $95 \%$ correct floor determination under the scenario in which $40 \%$ of RNs failed.
\end{abstract}

\section{Introduction}

Nowadays, there are several indoor location services available for a wide range of applications such as those in commercial, agriculture, medical, and military uses [1-3]. Wireless communications are the major driving force of the development of such indoor location services. Various wireless technologies can be employed for indoor positioning systems. Some systems make use of an existing wireless network infrastructure such as Wi-Fi [4-6], Bluetooth [7], and the visible light communication (VLC) [8]. More flexible and efficient systems employ IEEE 802.15.4 Wireless Sensor Networks (WSNs), providing advantages in terms of low power consumption, light weight, and low cost [9].

Existing indoor localization systems can be classified into three types based on the structure of the service areas. These include the indoor localization systems for two-dimensional service areas, three-dimensional service areas, and multistory buildings [10]. Most of the existing systems are designed for usage in two-dimensional areas where the position of a target object is specified by coordinates $(x, y)$ [10-12]. The second type of indoor positioning system considers a threedimensional space wherein the location of each target is derived from multiple coordinates $(x, y$, and $z$ ). The systems in the second type are usually applied in particular fields such as those in the industrial applications [13]. Recently, the indoor positioning systems for multistory buildings have gained more attention. Such systems need to specify not only coordinates $(x, y)$ but also the floor on which the object is located [14-18].

Most researches related to indoor positioning systems focus on either the development of the positioning techniques for improving the performance or the study and characterization of the unique properties of parameters such as the received signal strength (RSS) $[10,19]$. There are only a few works considering failure scenarios of RNs, which are 
the main equipment of the systems. In [20], the authors studied the robustness of the location determination when the system encountered a random failure of RNs. Under the $\mathrm{RN}$-failure scenarios, their experiment results showed that the positioning accuracy could drop from $10 \%$ to $50 \%$ when approximately $50 \%$ of RNs failed in the system. Other related works consider the robustness of the positioning systems against variation of RSSs [21] and changes to the service environments such as furniture [22] and service times [23].

Although some existing works have studied the degradation of the positioning performances due to RN failure and have considered the robustness of the systems against the changes of some parameters, the literature still lacks a floor determination algorithm that is robust in a situation when some RNs in the system fail. Such an algorithm is an important part of the localization technique for the complex indoor environment of the multistory building. Therefore, this paper aims to present a floor determination algorithm that can reliably identify the floor on which the object is located under various RN-failure scenarios. Our contributions are summarized as follows:

(i) We propose a floor determination algorithm which will serve as an important function of the indoor localization techniques for multistory buildings. The proposed algorithm is called the Robust Mean of Sum-RSS (RMoS) floor algorithm. It does not require the fingerprint database and can achieve the highest percentage of correct floor determination when compared to other existing techniques.

(ii) The proposed RMoS floor algorithm is robust against all of our tested $R N$-failure scenarios. It can work under either the fault-free scenario or the partial $R N$ failure scenarios. It can achieve greater than 95\% correct floor determination under the scenario in which $40 \%$ of RNs failed.

The remainder of this paper is organized into six sections as follows. In Section 2, we briefly summarize existing works on floor determination techniques. Section 3 provides an introduction to the $\mathrm{RN}$-failure scenario and explains our proposed robust floor determination algorithm. Section 4 describes the experimental environment, the wireless transceivers, and the setup parameters in this work. Section 5 presents the experimental results and discussion. Finally, Section 6 concludes the paper.

\section{Existing Floor Determination Techniques}

In wireless indoor positioning systems for multifloor buildings, the positioning error of a target within the two-dimensional plane might mean a false room or error distance of a few meters. On the other hand, the false floor determination could mean the incorrect detection of the target on a car parking floor instead of an office floor. Therefore, the problem of floor determination is another important issue for the positioning applications besides the target's coordinates.

$\mathrm{RN}$ failures during the online positioning phase cause RSS values to be missed. This can greatly affect the operations of the positioning systems such as the computational procedures and the signal sharing process in cooperative localization [24]. These failures can lead to accuracy performance degradation and a lack of system reliability [25]. Thus, a robust floor determination technique is required in order to achieve reliable indoor multifloor positioning systems that can provide high positioning accuracy under unexpected situations such as RN failures. In current literature, there are only a few papers considering the robustness of multifloor positioning systems, while the fault tolerance for the floor determination technique in terms of the presence of node failures has not been studied.

In $[16,17]$, the authors proposed floor determination algorithms that considered the movement of objects across the floors. In [16], the authors studied robots moving between floors by using incorporative information from the pressure sensor and the Wi-Fi access points (APs). Their floor determination algorithm is based on the RSS received from the APs that utilize a Maximum Likelihood (ML) to estimate the floor location of the robots. The authors in [17] presented the technique to estimate the robot position in each floor by using gyroscopes for recognizing the robot motion status on the stairs. However, these works only focused on the tracking of the floor changing when the robots moved in the staircase. Particularly, they did not consider the robustness in terms of faulty RNs during the online positioning phase. This reason has motivated our work to develop a floor determination algorithm to extend the capability of the indoor positioning systems to be able to handle situations where some RNs fail.

Existing floor determination approaches can be classified into two major types based on the use of the location fingerprint database: floor determination using the location fingerprint database and floor determination without the use of the location fingerprint database. For the approach that uses the fingerprint database, the system performance will depend on the number of the fingerprint locations which will require more steps and time for collecting the RSS data at the fingerprint locations in the offline phase [19]. In contrast, the approach that does not require the fingerprint locations has the advantage that it does not require the collection of the RSS data to create a database of floors in the offline phase. The system performance of the latter approach will depend on the calculation mechanism of the floor determination technique in the online phase only.

To evaluate the performance of the proposed floor determination algorithm, we compare our proposed algorithm with the other techniques in the literature, both those that use and those that do not use the location fingerprint database. The algorithms that use the fingerprint database in the floor determination process include the nearest floor algorithm [14] and Lui's floor algorithm [15]. Another algorithm that does not use the fingerprint database in the floor determination process is the group variance algorithm [14]. Brief descriptions of these three floor determination algorithms are presented as follows.

2.1. Nearest Floor Algorithm [14]. This algorithm is based on the K-Nearest Neighbor (KNN) algorithm. It requires 


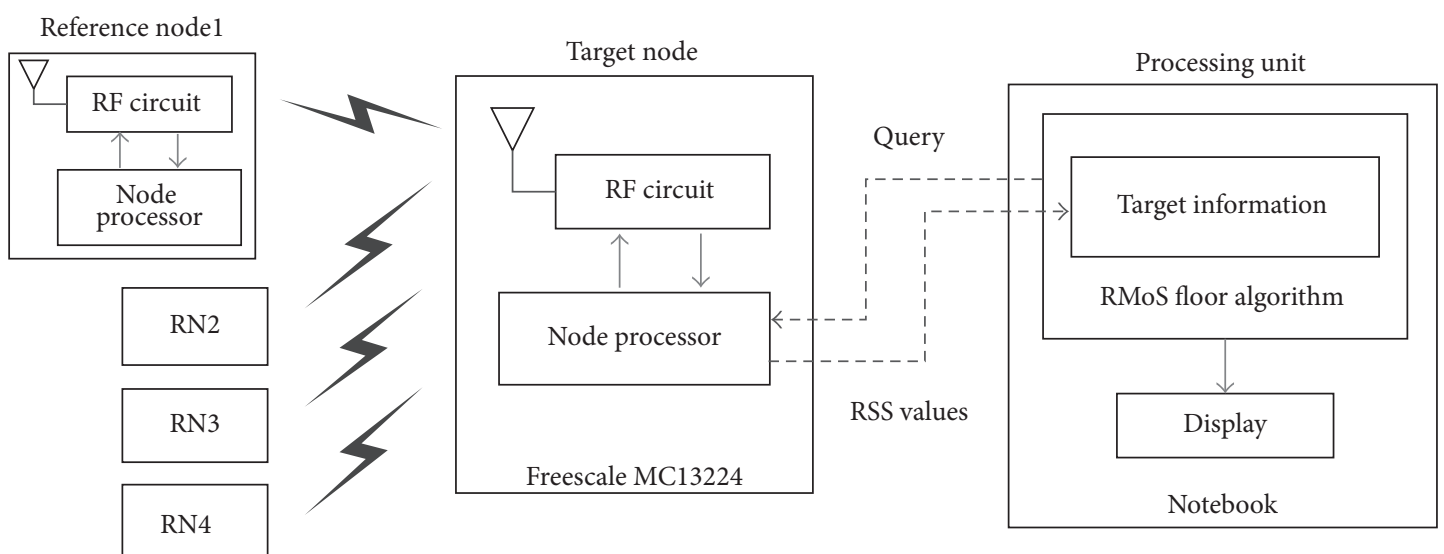

FIGURE 1: Block diagram of the main components in the indoor positioning systems.

collection of the RSSs in an offline phase in order to create the fingerprint database. This database records the information including the reference identification, the MAC address of the APs, the floor number, and the maximum RSS. To determine the floor number, the algorithm will compare the strongest online scanned RSS with the value in the fingerprint database. Then, the algorithm selects $k$ APs $(k=3)$ with the closest RSS values and applies the KNN algorithm to select the best matched floor number from the fingerprint database.

2.2. Lui's Floor Algorithm [15]. Lui's floor algorithm also requires the fingerprint database. This algorithm computes an average signal distance between the RSS value recorded in the fingerprint database and the online scanned RSSs. Then, the algorithm selects the floor on which the object is located from the minimum average signal distance.

2.3. Group Variance Algorithm [14]. This algorithm does not use the fingerprint database. It considers the distribution of the RSS values in each floor. It takes into account three online statistical parameters obtained from each AP, which consist of the range, the variance, and the availability. The algorithm adds weighted values based on the specified criteria to determine the floor scores, called floor points. After comparing the floor points, the algorithm selects the estimated floor number with the highest floor points.

\section{The RMoS Floor Algorithm}

This section describes our proposed floor determination algorithm for multistory buildings. First, in Section 3.1, the structure of the main components in the indoor positioning systems is described. Then, in Section 3.2, we describe our fault tolerance floor determination algorithm, which is called the Robust Mean of Sum-RSS (RMoS) floor algorithm and is enhanced from our previous floor determination technique presented in [18]. The RMoS floor algorithm does not require the fingerprint database, which consumes a lot of time for RSS data collection. Our proposed algorithm utilizes only the
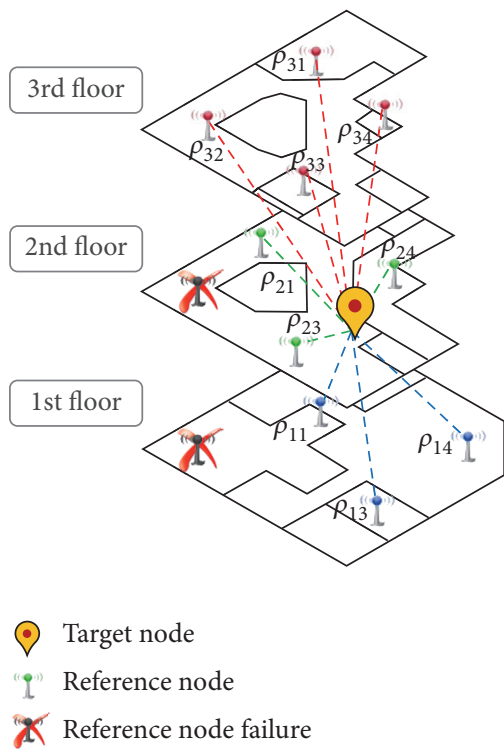

FIGURE 2: Floor determination schematic diagram.

online scanned RSS values received from the RNs in order to determine the floor location of the target node.

3.1. Structure of Indoor Positioning Systems. The main components of the indoor positioning systems used in this work are shown in Figure 1. They include the reference nodes (RNs), the target node, and the processing unit. The reference nodes are wireless transceivers that will send out signals upon request from the target node. The position of each reference node is known to the processing unit. The target node has a mobile wireless transceiver that helps determine on which floor number the target node is situated. The target node will query signals from the reference nodes. Then, it passes the signal information to the processing unit where the floor determination algorithm is executed.

Figure 2 illustrates an example of the structure of the indoor positioning system in a three-story building under the 
Input: Specify $\Gamma, F, N_{f}, x \%$, the initial value of $c$

Output: Floor number on which the target node is situated at the confidence level $c$

(1) The target node scans for RSS sent from the reference nodes.

(Begin the floor determination step)

(2) Repeat

(3) Repeat

(4) Compute the RSS summation $\gamma_{f}^{\tau}=\sum_{n \in \Re_{f}} \rho_{f n}^{\tau}$

(5) Until $\tau=\Gamma$

(6) Set $\Lambda_{f}=\left\{\gamma_{f}^{1}, \gamma_{f}^{2}, \ldots, \gamma_{f}^{\tau}, \ldots, \gamma_{f}^{\Gamma}\right\}$

(7) Until $f=F$

(8) Set the confidence level $c=$ the specified initial value

(9) Calculate $\Phi\left(\Lambda_{f}\right)$ with \%CI $=c$ for all $f \in \Psi$

(10) If $\Phi\left(\Lambda_{f}\right)$ of any floor is overlapped

(11) Repeat

(12) Reduce the confidence level $c$

(13) Calculate $\Phi\left(\Lambda_{f}\right)$ with $\% \mathrm{CI}=c$ for all $f \in \Psi$

(14) Until no overlap of $\Phi\left(\Lambda_{f}\right)$

(15) $\quad c$ is the \%CI of sum of the strongest RSS from $\mathfrak{R}_{f}$ for all $f \in \Psi$

(16) The floor where the target node is located is associated with the floor that has the highest $\Phi\left(\Lambda_{f}\right)$ at the confidence level $c$. floor $=\arg \max _{f}\left(\Phi\left(\Lambda_{f}\right)\right)$ at the confidence level $c$

Algorithm 1: Pseudo code for RMoS floor algorithm.

RN-failure scenario in which two RNs fail. In this diagram, four RNs are installed on each floor. Dashed lines represent RSSs that the target node receives from all RNs.

In Figure 2, one RN on the 1st floor and another RN on the 2nd floor become unavailable. This may be caused by hardware failures, software errors, or other faults. Hence, the online scanned RSSs of the target node would not have signals from these RNs.

3.2. Framework of RMoS Floor Algorithm. Our proposed algorithm determines the floor number of the target node based on the summations of the online scanned RSSs that the target node received from the working $\mathrm{RNs}$ in the building. In order to handle the fluctuation nature of the indoor wireless signal, the concept of the confidence interval comparison [26] is adopted in the RMoS floor algorithm. Specifically, RMoS compares the confidence intervals for the mean of the RSS summations. The following describes how the RMoS floor algorithm works.

The following list defines the parameters used in our proposed algorithm. Algorithm 1 describes the pseudo code of the RMoS floor algorithm. The procedures are as follows. First, the target node scans for the RSSs sent from RNs installed in the multifloor building. A set of sampled RSS values and the reference node IDs of each RSS value are recorded as the input data vector for the floor determination step. During the RSS scanning procedure, the signal from each $\mathrm{RN}$ is scanned and recorded for $\Gamma$ samples. The number of samples $(\Gamma)$ is selected such that it is not too small that it becomes insignificant in the statistics and it is not too large that it would require too much time to collect the data. The sampling rate depends on the capability of the wireless transceiver to respond to the signal query. In the case that the number of samples is less than 30 , the $t$-distribution is applied in the confidence interval calculation [26]. Otherwise, the normal distribution should be utilized [26].

\section{List of the Parameter Definitions}

$\Lambda_{f}$ : a set of the summations of the strongest RSSs on the $f$ th floor $\left\{\gamma_{f}^{1}, \gamma_{f}^{2}, \ldots, \gamma_{f}^{\Gamma}\right\}$

$\Psi$ : a set of floors in the service areas $\{1,2, \ldots, F\}$

$\mathfrak{R}_{f}$ : a set of $x \%$ of RNs on the $f$ th floor that gives the strongest RSS values

$x \%$ : the percentage of RNs that would be considered on each floor in the floor determination step

$\Phi(\cdot)$ : the confidence interval for the mean of the RSS summations

$\gamma_{f}^{\tau}$ : the summation of the RSSs that the target node receives from $\mathfrak{R}_{f}$

$\rho_{f n}^{\tau}$ : the RSS value which is $\tau$ th measurement from $n$th $\mathrm{RN}$ on the $f$ th floor

$N_{f}$ : maximum number of RNs on the $f$ th floor

$\Gamma$ : the number of RSS samples

$F$ : the total number of floors in the multistory buildings

$L_{f}$ : the lower limit of the confidence of the population mean on the $f$ th floor

$U_{f}$ : the upper limit of the confidence of the population mean on the $f$ th floor

$\mu$ : the population mean 
$\sigma^{2}:$ the sample standard deviation

$\eta$ : the sample mean

$c$ : the confidence level (i.e., $95 \%$ or $90 \%$ )

$\beta$ : a significance level (i.e., 0.05 or 0.1 )

$1-\beta$ : the confidence coefficient

$t_{[1-(\beta / 2) ; \Gamma-1]}$ : the $(1-(\beta / 2))$-quantile of $t$-distribution and $\Gamma$ samples

In the floor determination step, first the RSS summation $\gamma_{f}^{\tau}$ is computed by using (1). For the $\tau$ th sampling, $\gamma_{f}^{\tau}$ is the summation of RSSs received from RNs in $\mathfrak{R}_{f}$, where $\mathfrak{R}_{f}$ is the set of $x \%$ of RNs on the $f$ th floor that gives the strongest RSS values. The variable $\rho_{f n}^{\tau}$ denotes the online scanned RSS which is scanned in the $\tau$ th sampling and it is the signal received from $n$th $\mathrm{RN}$ which is on the $f$ th floor. Note that $n=1,2, \ldots, N_{f}$, where $N_{f}$ is the maximum number of RNs on the $f$ th floor. $\Psi$ is the set of floors in the service areas.

$$
\gamma_{f}^{\tau}=\sum_{n \in \Re_{f}} \rho_{f n}^{\tau} .
$$

Next, a set $\Lambda_{f}=\left\{\gamma_{f}^{1}, \gamma_{f}^{2}, \ldots, \gamma_{f}^{\tau}, \ldots, \gamma_{f}^{\Gamma}\right\}$ is obtained. $\left(\Lambda_{f}\right.$ is a set of the RSS summations on $f$ th floor for $\Gamma$ number of RSS samples.) After that $\Phi\left(\Lambda_{f}\right)$, which is the confidence interval $\left(L_{f}, U_{f}\right)$ for the mean of the RSS summations on each floor, is calculated by using

$$
p\left(L_{f} \leq \mu \leq U_{f}\right) \approx 1-\beta,
$$

where $L_{f}$ is the lower limit, $U_{f}$ is the upper limit, and $1-\beta$ is the probability that the population mean of RSSs $(\mu)$ will lie between $L_{f}$ and $U_{f}$. They are calculated by using (3) to (5), respectively.

$$
\begin{aligned}
L_{f} & =\eta(\Gamma)-t_{[1-(\beta / 2) ; \Gamma-1]} \sqrt{\frac{\sigma^{2}(\Gamma)}{\Gamma},} \\
U_{f} & =\eta(\Gamma)+t_{[1-(\beta / 2) ; \Gamma-1]} \sqrt{\frac{\sigma^{2}(\Gamma)}{\Gamma}}, \\
\beta & =\frac{1}{2}\left(1-\frac{c}{100}\right) .
\end{aligned}
$$

Note that (3) and (4) are used to determine the lower limit $L_{f}$ and upper limit $U_{f}$ of the sample values of the RSS summations at $c \%$ confidence level. $\eta$ refers to the sample mean of RSS summations. $\sigma^{2}$ is the sample standard deviation and $t_{[1-(\beta / 2) ; \Gamma-1]}$ is the $(1-(\beta / 2))$-quantile of $t$-distribution and $\Gamma$ samples [26].

Finally, to determine the floor number where the target node is situated, the proposed RMoS floor algorithm compares the confidence intervals $\Phi\left(\Lambda_{f}\right)$ over all floors in $\Psi$. Then, RMoS selects the floor number that has the highest value of $\Phi\left(\Lambda_{f}\right)$ as the floor where the target node is situated.

\begin{tabular}{|c|c|}
\hline Parameter & Detail \\
\hline Floor dimensions & $\begin{array}{l}35 \mathrm{~m} . \times 35 \mathrm{~m} \text { (Building A), } 75 \mathrm{~m} \times 75 \mathrm{~m} \\
\text { (Building B) }\end{array}$ \\
\hline RN placement & Uniform placement \\
\hline Test points & Total of 75 test points (15 test points/floor) \\
\hline Number of RNs & Total of 20 nodes ( 4 nodes/floor) \\
\hline Test scenario & Fault-free and RN failures \\
\hline $\mathrm{RN}$ failures pattern & $\begin{array}{l}20 \text { failure patterns are random for each } \mathrm{RN} \\
\text { failure scenario }\end{array}$ \\
\hline
\end{tabular}
This can be written in

$$
\text { floor }=\arg \max _{f}\left(\Phi\left(\Lambda_{f}\right)\right) .
$$

TABLE 1: Setup parameters.

\section{Experimental Setups}

The performance of the proposed RMoS floor algorithm is evaluated through extensive experiments and the results are compared with other existing floor algorithms. In particular, the results are compared with two approaches of floor determination algorithms. The first approach uses the fingerprint database in the floor determination process. The existing floor determination algorithms in this approach that are compared with our proposed algorithm include the nearest floor algorithm [14] and Lui's floor algorithm [15]. The other approach compared here does not use the fingerprint database in the floor determination process. The existing algorithm in this approach that is compared with our proposed algorithm is the group variance algorithm [14]. The reason that we compare our proposed algorithm with the two approaches (with/without fingerprint database) is to evaluate and analyze how each approach performs in different network scenarios.

In our experimental study, two five-story buildings with different floor structures and with different dimension areas are tested. The first building, labelled Building A, is a library with dimensions of approximately $35 \mathrm{~m}$ (width) $\times 35 \mathrm{~m}$ (length). The second building, labelled Building $\mathrm{B}$, is an office building with dimensions of about $75 \mathrm{~m}$ (width) $\times 75 \mathrm{~m}$ (length). The floor layouts of both buildings are illustrated in Figures 3 and 4, respectively. Twenty RNs were deployed in both service areas. Four RNs were installed uniformly on each floor as shown in Figures 3(b) and 4(b). Fifteen test points were randomly selected on each floor by using the uniform random function of MATLAB (i.e., a total of 75 test points) to analyze the performance of the floor determination algorithms. The setup parameters for the experiment are summarized in Table 1.

In order to handle the situations of signal unavailability due to RN failure, we did not use RSS values received from all RNs in the system for the summation of RSSs, but instead only the strongest RSS values received from all RNs located on each floor are considered. From preliminary testing, we found that considering which $50 \%$ of RNs on each floor give the strongest RSS values (i.e., $x=50 \%$ ) is suitable for the RSS summations under $\mathrm{RN}$ failure.

Figure 5 illustrates the experimental equipment installed on each floor. This includes four RN nodes which are placed at a height of $2 \mathrm{~m}$. The target node is connected to a computer 


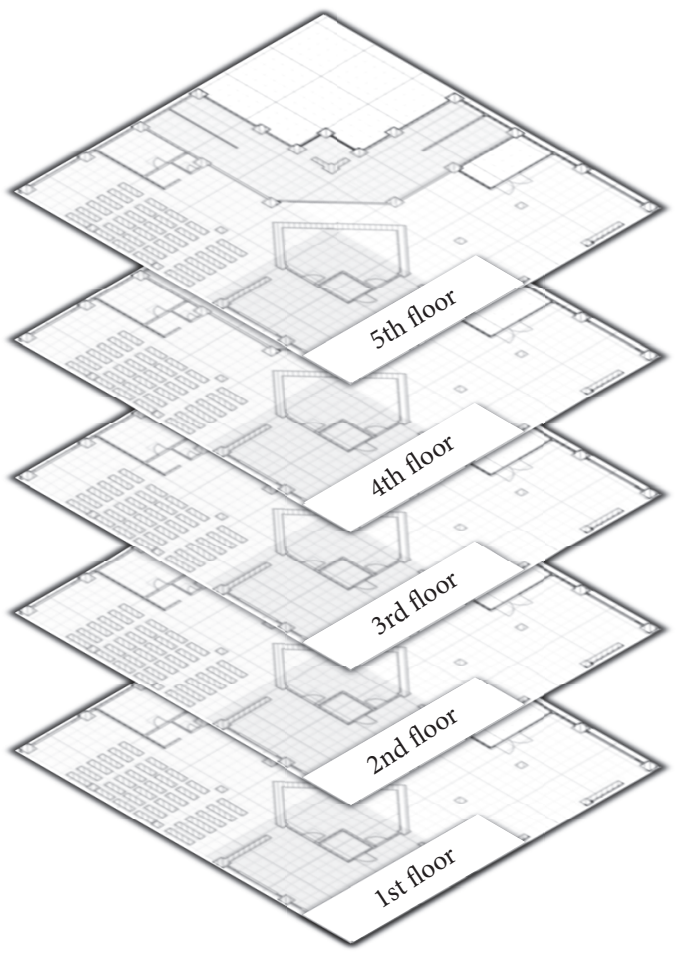

(a)

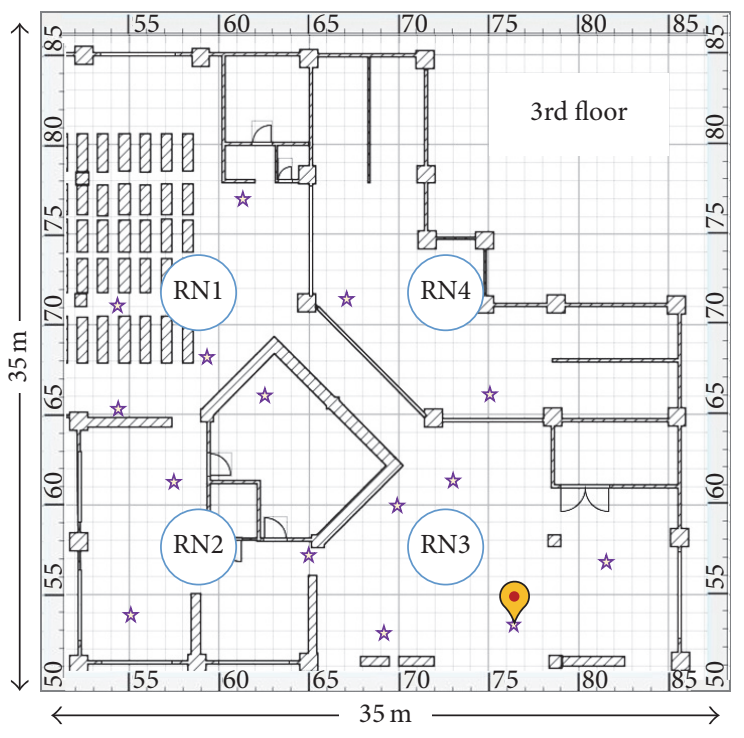

The 44th test point

RN Reference node

岁 Test point

(b)

Figure 3: Building A. (a) The five-story structure. (b) Floor plan and example of 15 test points labelled with stars on the 3rd floor.

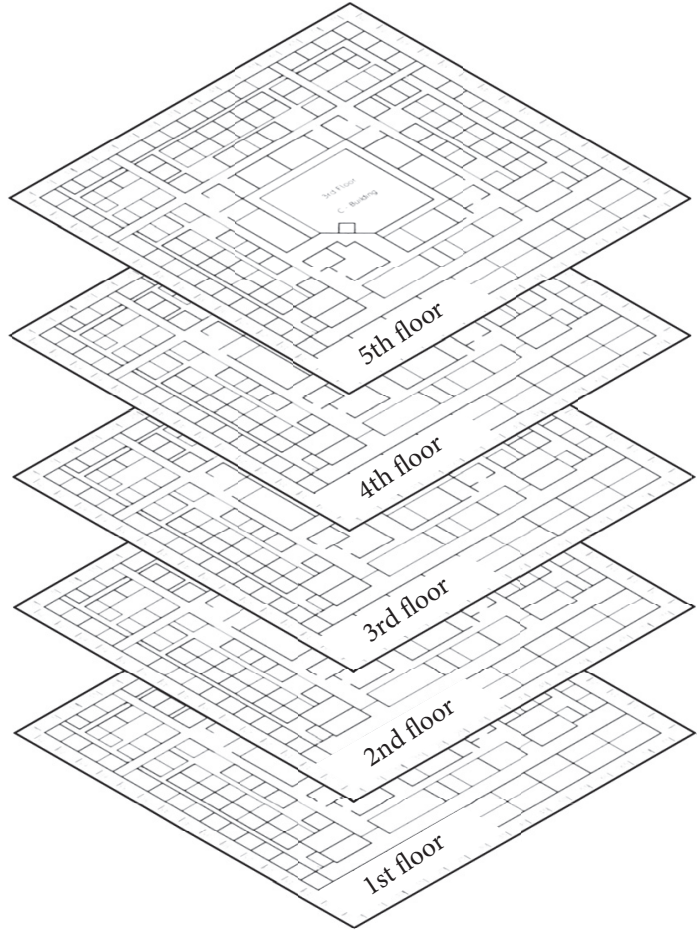

(a)

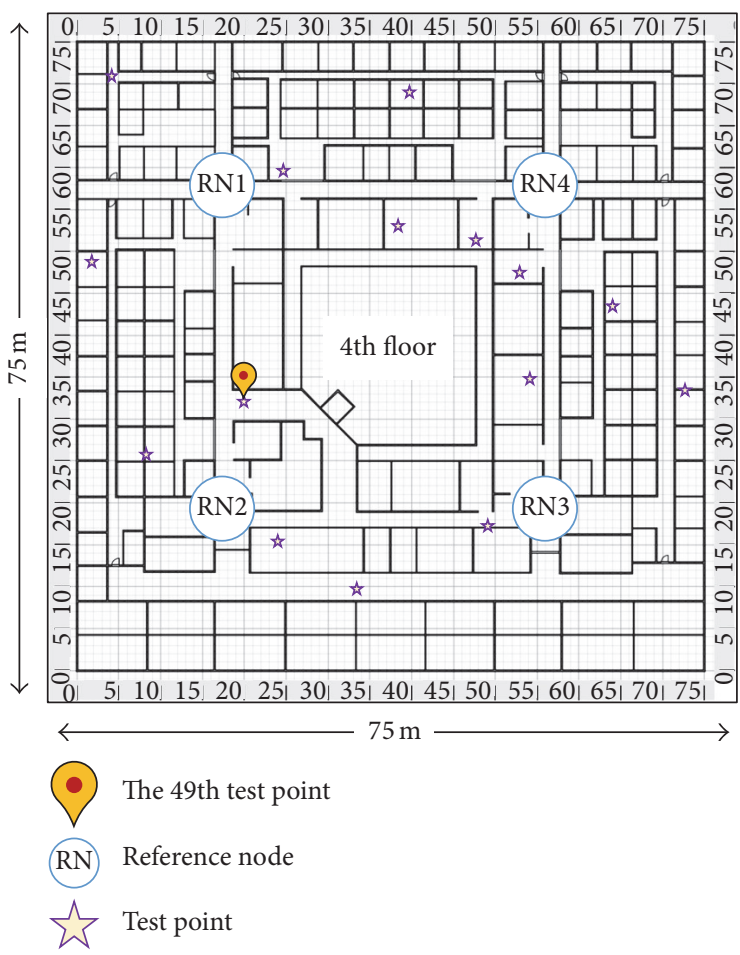

(b)

Figure 4: Building B. (a) The five-story structure. (b) Floor plan and example of 15 test points labelled with stars on the 4 th floor. 


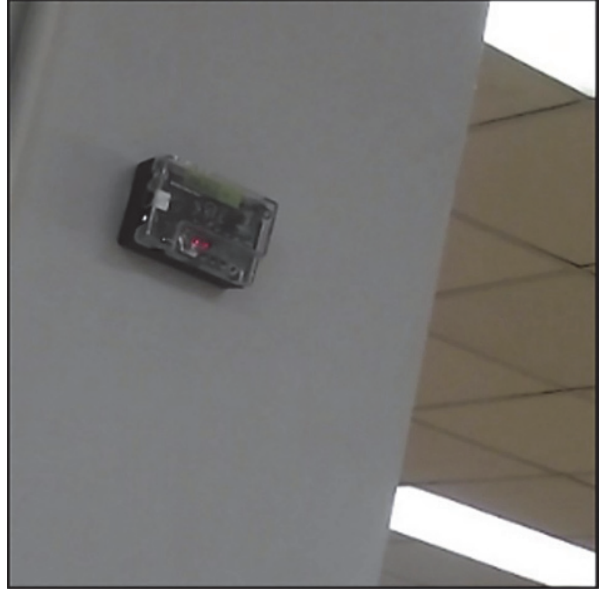

(a)

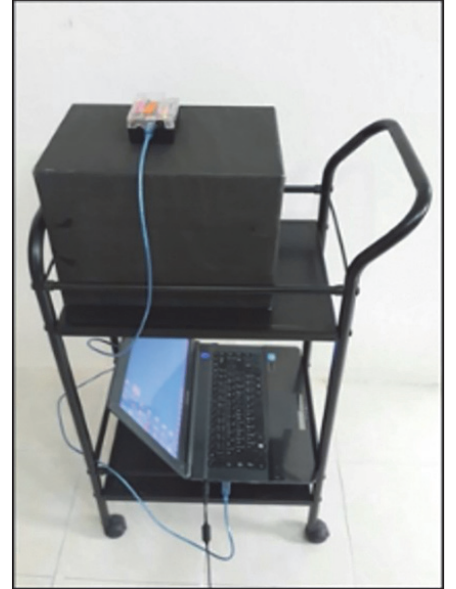

(b)

FIgURE 5: Experimental equipment in this work. (a) Reference node. (b) Target node.

TABLE 2: Specifications of the wireless transceivers.

\begin{tabular}{lc}
\hline Specification & Detail \\
\hline Manufacturer & Freescale \\
Chipset & MC13224V \\
Frequency range & $2.405 \mathrm{GHz}-2.480 \mathrm{GHz}$ \\
Operating channel & $\mathrm{CH} 26(2.480 \mathrm{GHz})$ \\
Rx sensitivity & $-95 \mathrm{dBm}$ \\
Transmit power & $+3 \mathrm{dBm}$ \\
Antenna & Inverted F-antenna \\
\hline
\end{tabular}

notebook on which the floor determination algorithm is executed. The height of the target node is $0.8 \mathrm{~m}$. IEEE 802.15.4 wireless transceivers were deployed in this work. They have Freescale MC13224V third-generation chipsets with builtin ARM7TDMI processors. The antennas of the wireless transceivers are the inverted F-shape antennas and different sets operate at $2.480 \mathrm{GHz}$ (i.e., channel 26 of IEEE 802.15.4 standard). This is to avoid or minimize the interference from Wi-Fi networks in the areas. The target node will gather the RSS values that are transmitted from the RNs. Those measured RSS values are used to calculate the floor number of the target node. The sampling rate of wireless transceivers in this work is 1 sample in 3 seconds. Note that we consider the stationary node in our experimental study. Table 2 shows specifications of the wireless transceivers used in our experiments.

Two different scenarios were considered in our experiments. The first scenario is a fault-free scenario in which all RNs worked properly. The second scenario is $R N$-failure scenario in which some RNs in the system fail. In particular, we randomly turned off two and eight RNs in the system under the condition that the number of failed RNs on each floor did not exceed $50 \%$ of the total number of RNs on that floor (i.e., in this study, the maximum number of failed RNs on each floor is two nodes).

\section{Results and Discussion}

5.1. Fault-Free Scenario. The experiments in this scenario consider that all RNs in the service area work properly. The study is organized into two sets of experiments. First, Section 5.1.1 aims to analyze the characteristics of the summation of RSSs that the target node receives from RNs installed on each floor. Then, Section 5.1.2 aims to compare the performance of the proposed algorithm with that of other existing floor determination techniques in the case of no $\mathrm{RN}$ failure.

5.1.1. Analysis of RSS Summation Characteristics. First, we analyze the characteristics of the RSS summations at a particular point. Consider the 44th test point located on the third floor of Building $\mathrm{A}$ and consider the 49th test point located on the fourth floor of Building B as indicated by the yellow symbol in Figures 3(b) and 4(b), respectively. In both cases, $F=5, N_{f}=4, x=50 \%$, and $\Gamma=20$. (Note that it would take approximately one minute to collect 20 samples.)

From all RNs in the online RSS system, we recorded 20 samplings that were received at the 44th and 49th test points. For each sampling $\tau$ th, 20 RSS values received from all RNs in the system were recorded and denoted by $\rho_{f n}^{\tau}$ ( $f$ represents the floor number of the $n$th RN that transmitted a particular RSS value.) Then, $\gamma_{f}^{\tau}$ (the summation of the strongest RSSs received from $50 \%$ of RNs located on the $f$ th floor for the $\tau$ th sampling) was calculated by using (1). Finally, we obtained 100 values of the RSS summations for 20 signal samplings (i.e., for each $\tau$ th sampling, $\gamma_{1}^{\tau}, \gamma_{2}^{\tau}, \ldots, \gamma_{5}^{\tau}$ were computed).

Figures 6(a) and 6(b) are the histogram plots of the RSS summations at the 44th test point of Building $\mathrm{A}$ and the 49th test point of Building B, respectively. In the histogram, each color represents a summation of RSSs received from RNs located on each floor. In the case of the 44th test point of Building A, we can observe from Figure 6(a) that the RSS summations of the green bins (the summation of RSSs received from RNs on the third floor) are greater than those 


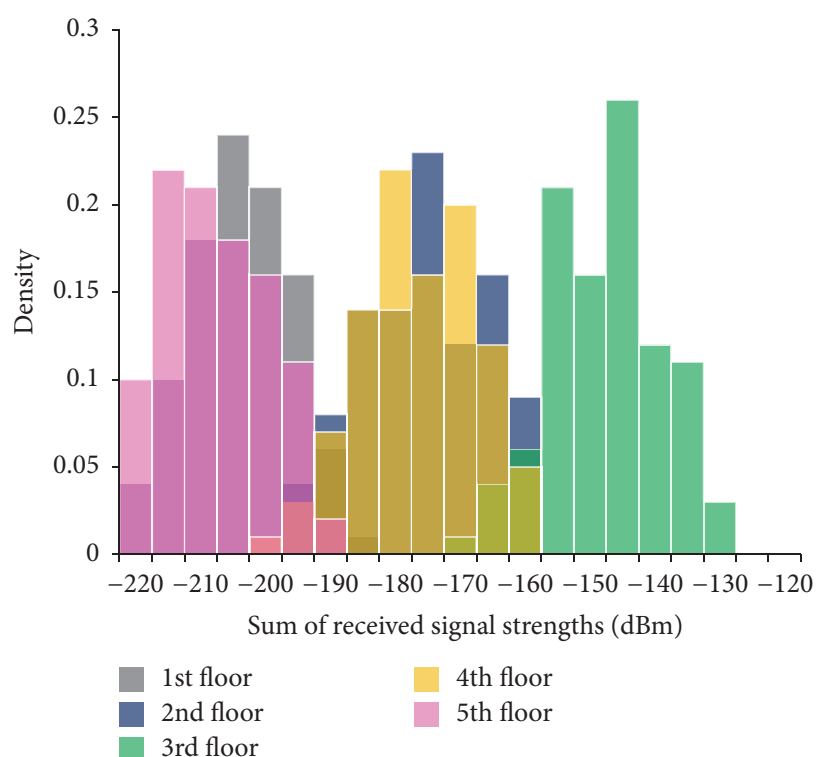

(a)

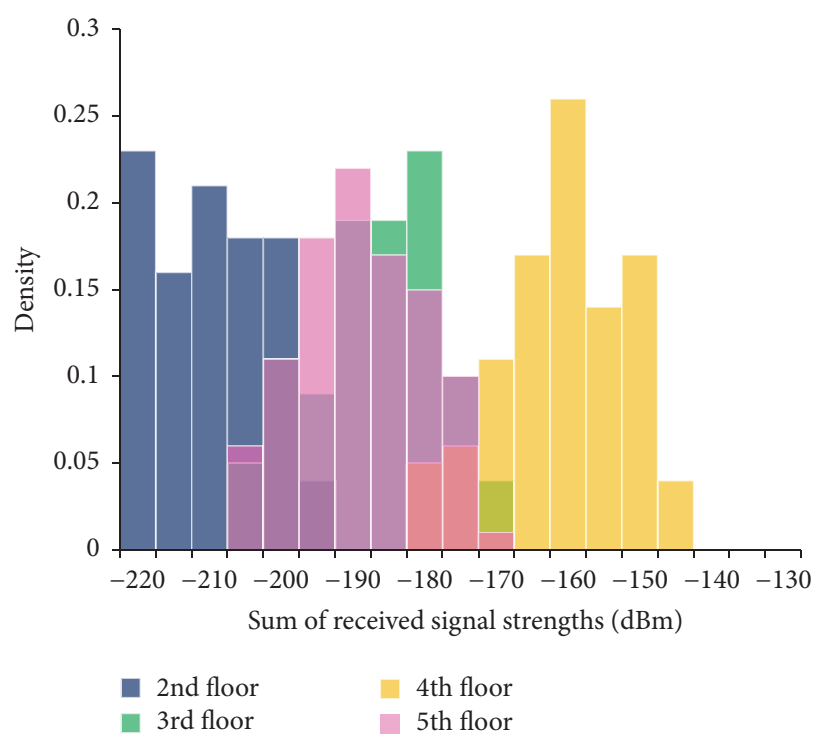

(b)

FIGURE 6: Histogram of the RSS summation in the fault-free scenario at (a) the 44th test point located on the third floor of Building A and (b) the 49 th test point located on the fourth floor of Building B.

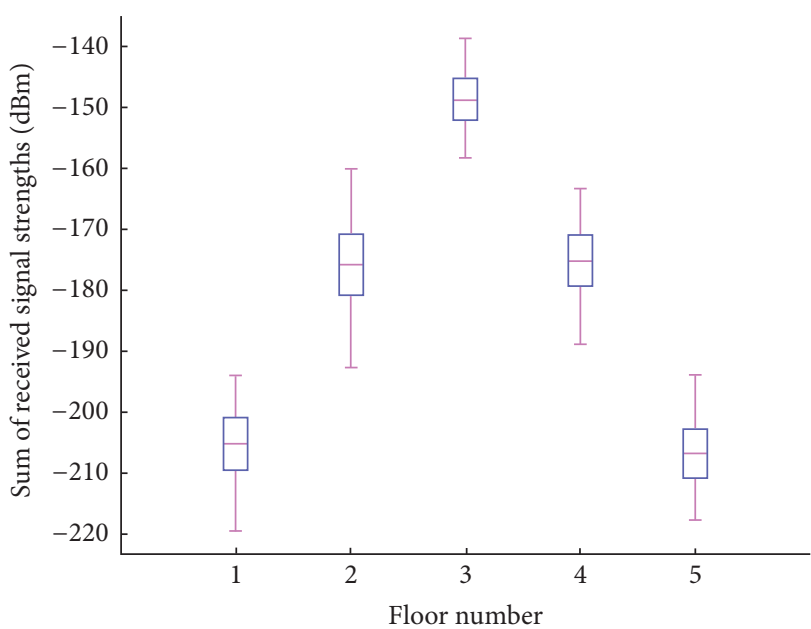

(a)

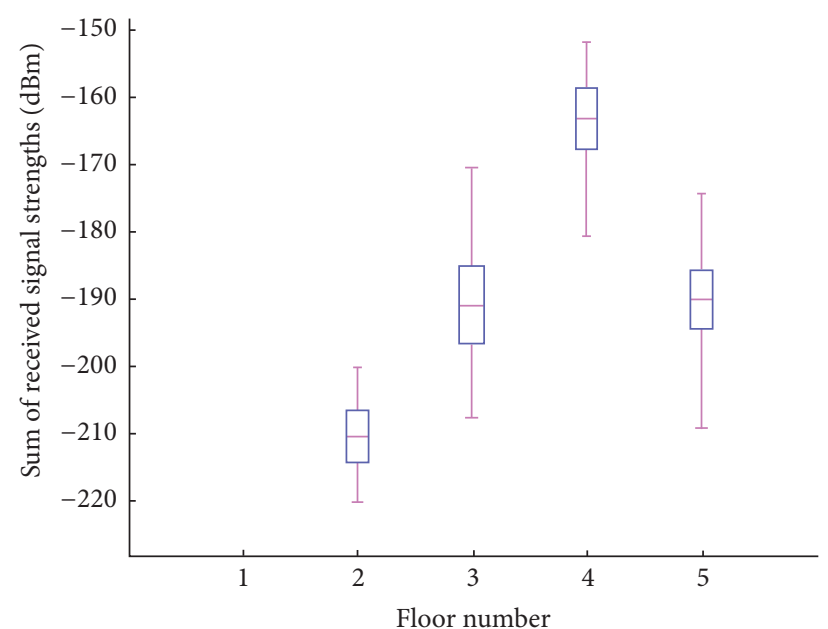

(b)

FIGURE 7: 95\% confidence interval for the mean of the RSS summations in the fault-free scenario at (a) the 44th test point located on the third floor of Building A and (b) the 49th test point located on the fourth floor of Building B.

of the other bins. This indicates that the summations of RSSs received from RNs located on the third floor (which is the floor where the test point was located) are stronger than those received from RNs located on the other floors. The same results can be observed in the case of the 49th test point of Building B (Figure 6(b)) in which the RSS summations of the yellow bins are the strongest. This indicates that the 49th test point is located on the fourth floor. Note that in Figure 6(b) there is no bin of the summation of RSSs received from RNs on the first floor because signals from RNs on the first floor could not reach the 49th test point, which was located on the fourth floor. These characteristics of the RSS summations observed from this study are applied in our proposed RMoS floor algorithm as described in Section 3.

Figures 7(a) and 7(b) illustrate a 95\% confidence interval for the mean of the RSS summations on each floor for Building A and Building B, respectively. The confidence interval is shown by a box stretching between the lower and upper confidence limits. The pink line in the box indicates the sample mean. From Figures 7(a) and 7(b), it is clear that the confidence interval for the mean of the RSS summations on the actual floor where the target node was located is higher than that of the other floors (i.e., $\Phi\left(\Lambda_{3}\right)$ and $\Phi\left(\Lambda_{4}\right)$ are highest for the case of Building $A$ and Building $B$, resp.). In 
both cases, there is no overlap between the highest confidence intervals and the rest of the confidence intervals. This shows that the sample values of the RSS summations on both target floors are significantly higher than those of the other floors at a 95\% confidence level. Therefore, the proposed RMoS floor algorithm correctly reports that the target node is on the third floor for Building A and the fourth floor for Building B. Note that in Figure 7(b) there is no confidence interval of the mean of the RSS summations on the first floor because the target node could not receive a signal from the RNs on the first floor.

\subsubsection{Performance Comparisons under Fault-Free Scenario.} This section aims to compare the proposed RMoS floor algorithm with other existing techniques under the normal operating scenario where all RNs work properly. In particular, the proposed algorithm was compared with the nearest floor algorithm [14], the group variance algorithm [14], and Lui's floor algorithm [15]. The performance of the floor determination algorithms was evaluated by the percentage of the correct floor determination. The experiments were set up as described in Section 4. Additionally, to compare the $\mathrm{RMoS}$ floor algorithm with the other algorithms that use the fingerprint database, we consider the effects of using different numbers of fingerprint locations. In particular, three different settings of fingerprint locations were considered: 10,30 , and 50 locations. They were selected uniformly across the service area.

Figures 8(a) and 8(b) compare the percentages of the correct floor determination among four floor determination algorithms for Building A and Building B, respectively. The results show that the proposed RMoS floor algorithm outperforms the other three algorithms and yields a 100\% correct floor determination for all cases of the fingerprint setting in both buildings. The reason is that RMoS does not use the fingerprint database in the floor computing procedures but it utilizes only the online RSSs that the target node receives from RNs in the building. The RMoS floor algorithm determines the floor number based on the value of the confidence interval of the RSS summations as demonstrated in Section 5.1.1.

The nearest floor algorithm and Lui's floor algorithm, on the other hand, use the fingerprint database in the floor determination process. Consequently, from Figure 8 , it can be observed that the performances of the nearest floor algorithm and Lui's floor algorithm are dependent on the number of fingerprint locations, whereby a higher number of fingerprint locations yield higher percentages of correct floor determination. The nearest floor algorithm yields the lowest percentage of correct floor determination at $24 \%$ and $25.3 \%$ for the case of 10 fingerprint locations for Building $\mathrm{A}$ and Building B, respectively. However, the nearest floor algorithm in the case of Building B was able to obtain a 100\% correct floor determination performance at the price of using 50 fingerprint locations.

The reason that the nearest floor algorithm and Lui's floor algorithm perform worse when using lower numbers of fingerprint locations is that there is a limitation in these floor determination techniques that use the location fingerprint database. That is, the location fingerprinting approach requires a site survey and collection of RSS patterns from all RNs installed in the service area during the offline phase. If the system designer collected insufficient fingerprint locations (i.e., low fingerprinting granularity), this will result in a low resolution location fingerprint database that cannot represent the RSS characteristics of the service area. Therefore, the performance of the floor determination algorithms that use a poor resolution fingerprint database will be degraded during the online phase. This will result in lower accuracy in determining the correct floor.

On the other hand, even though these floor determination algorithms can increase the number of fingerprint locations in their database and thus can improve the floor determination accuracy, this will be achieved at the cost of longer fingerprint collection time during the offline phase. This can result in weeks spent on site survey and data collection for a large service area. Moreover, if there are any changes in the building structure or layout such as the relocation of furniture or an increase or decrease in the number of RNs in the system in the fingerprint database, the location fingerprint data will have to be collected again.

Considering the group variance algorithm which does not use the fingerprint locations, it has a constant percentage of correct floor determination in all cases of the fingerprint locations. From Figures 8(a) and 8(b), we can observe that the performance of the group variance algorithm depends on the floor size of the building; a bigger floor size yields a better performance. The percentage of correct floor determination of the group variance algorithm of Building B (with a floor size of $75 \mathrm{~m} \times 75 \mathrm{~m}$ ) is higher than that of Building A (with a floor size of $35 \mathrm{~m} \times 35 \mathrm{~m}$ ), that is, $93.3 \%$ for Building B and $61.3 \%$ for Building A.

The reason that the results of the group variance algorithm in a small building are worse than those in a large building which has the same number of RNs is the error in calculation of floor points. This is the result of the similarity of RSSs inside the building. For example, when considering the floor points which were calculated from the variance of signals in a small building, we can observe that the RSSs received from RNs at the object location at the actual target floor and the adjacent floor are very similar. Therefore, the variance of the adjacent floor may be selected as the highest variance instead of the variance of the actual target floor. This is the reason that the floor points are incorrect and cause the group variance algorithm to determine the wrong floor.

On the other hand, in the case of a large building, the RSS received from RNs on different floors at the target node location may be small or may not be present because of the large distance and higher attenuation of the signals. Therefore, the floor that has the highest variance will be the one that receives the most signals from the RNs and will be the floor on which the target node is located. The algorithm can determine the floor correctly. In other words, the RSSs within the large building can be distinguished easily and have a positive effect on the accuracy of the group variance algorithm.

5.2. RN-Failure Scenarios. In this section, the experimental setup is the same as that for those described in Section 4. 


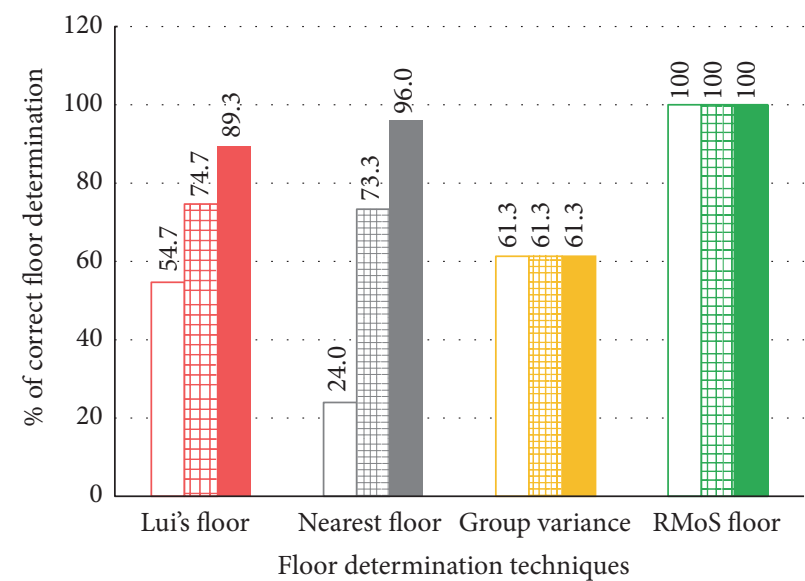

10 fingerprints 30 fingerprints 50 fingerprints

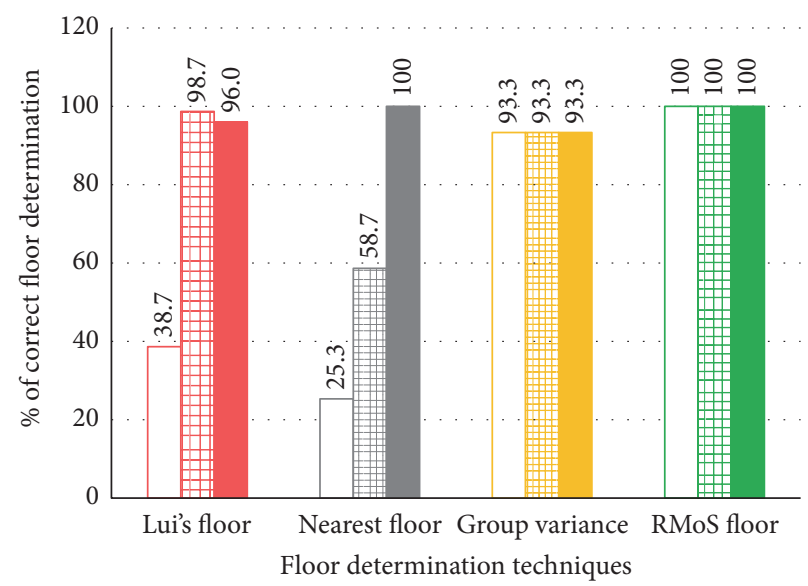

10 fingerprints 30 fingerprints 50 fingerprints

(a)

(b)

FIGURE 8: Percentage of correct floor determination of the fault-free scenario at (a) Building A and (b) Building B.

Additionally, however, this section considers that there are some faulty RNs in the system. In particular, two cases of $\mathrm{RN}$ failure were considered, including 2-RN failure and 8-RN failure. The experimental results of each case of RN failures were averaged from twenty faulty patterns. For example, in the case of 2-RN failure, we randomly selected and turned off two RNs from all RNs to create twenty patterns of two failed RNs. For each pattern, we obtained the percentage of correct floor determination from 75 test points selected uniformly throughout the whole building. Then, an average of the percentages of correct floor determination of those twenty patterns of 2-RN failure was computed. For the case of 8-RN failure, the same procedures were conducted.

To evaluate the performance of the existing floor determination algorithms that use and that do not use the fingerprint database under the RN-failure scenarios and to observe the effects of the fingerprint locations, five different settings of fingerprint locations were considered: 50, 40, 30, 20, and 10 locations. They were selected uniformly across the service area.

Figures 9(a) and 9(b) compare the average percentages of correct floor determination under three scenarios: faultfree, $2-\mathrm{RN}$-failure, and 8-RN-failure scenarios. The results are represented by a dotted line with triangle markers, a dashed line with cross markers, and a solid line with dot markers, respectively.

Considering the results of the nearest floor and Lui's floor algorithms which use the fingerprint database in the floor determination process, the performances of both algorithms are affected by the faulty RNs. For both the 2-RN-failure and 8 -RN-failure scenarios, the trend of the performance is the same as that of the results in the case of the fault-free scenario presented in Section 5.1.2 in which the performances of both algorithms are dependent on the number of fingerprint locations; using a larger number of fingerprint locations results in higher percentages of correct floor determination. For example, considering the case of Building A under the 2-RN-failure scenario (Figure 9(a)), the percentages of correct floor determination of the nearest floor algorithm decrease from $94.8 \%$ to $91.1 \%$ to $68.5 \%$ to $20.6 \%$ to $19.8 \%$ when using the number of 50, 40,30, 20, and 10 fingerprint locations, respectively. As the number of failed RNs increases to eight (i.e., 8-RN-failure scenario), the performance of the nearest floor algorithm is worse than that in the 2-RN-failure scenarios. It can be observed that when using a large number of fingerprint locations, RN failure affects the performance of the nearest floor algorithm more than when using a small number of fingerprint locations. The reason for this is that the nearest floor algorithm and Lui's floor algorithm have an error in their RSS matching processes in which the number of discovered RNs at the target node location during the online phase must be equal to the number of RNs in the database (radio map). Therefore, the missing RSS component(s) due to $\mathrm{RN}$ failure during the online phase/location determination will cause errors in the matching processes. A larger number of RN failures, such as 8 nodes out of 20 nodes, will have a larger impact than when only 2 nodes out of 20 nodes have experienced a failure. Similar results were obtained for the case of Building B (Figure 9(b)).

Considering the group variance algorithm which does not use the fingerprint database, its performance is also affected by the RN failure. In the case of Building A under the 2-RN-failure and 8-RN-failure scenarios (the yellow lines in Figure 9(a)), the percentage of correct floor determination drops from $61.3 \%$ to $55.8 \%$ and $47.7 \%$, respectively. Similar effects can be observed in the case of Building B (the yellow lines in Figure 9(b)). The reason that increasing the number of $\mathrm{RN}$ failures degrades the performance of the group variance 


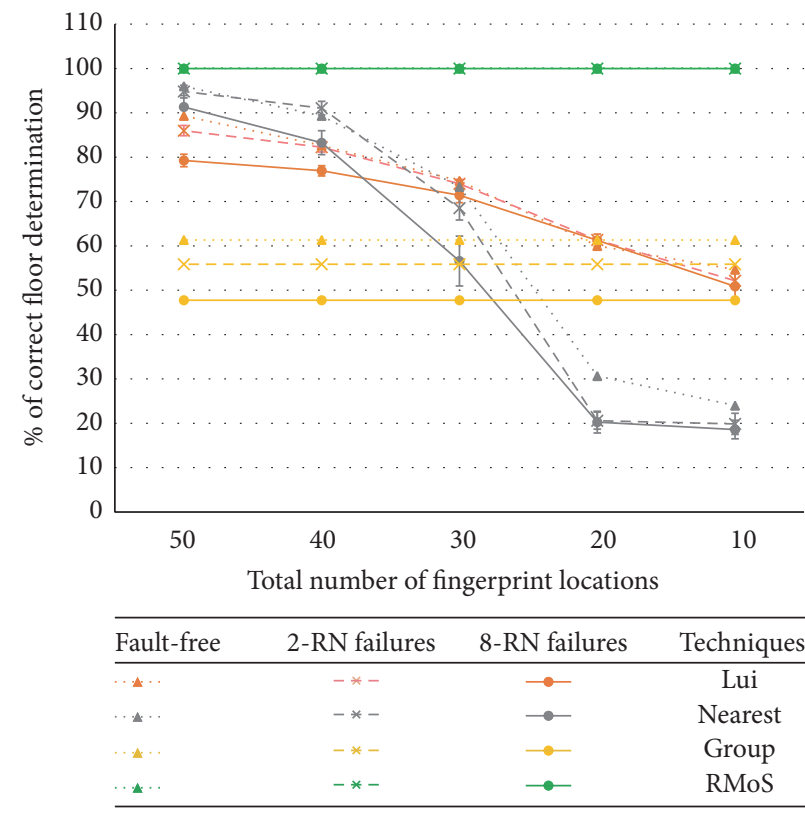

(a)

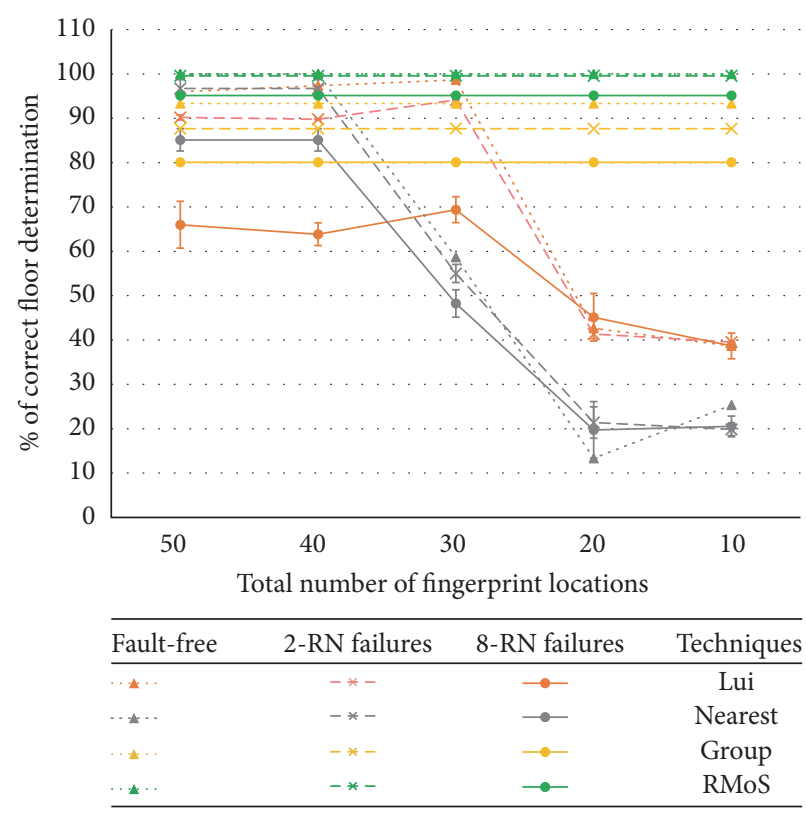

(b)

FIGURE 9: Percentage of correct floor determination in fault-free, 2-RN-failure and 8-RN-failure scenarios at (a) Building A and (b) Building B.

is the change in the calculation of three online statistical parameters obtained from each RN. The missing RSSs on some floors will affect the calculation of floor points and cause errors. For instance, the calculation of floor points is obtained from the ranges that consider the maximum and minimum value of RSSs at the target node from RNs on each floor. If the failure occurs to RN which had the largest RSSs on that floor, the range which was calculated on that floor will be incorrect. For this reason, the increasing number of failed RNs will result in the poorer performance of the group variance algorithm.

Considering the RMoS floor algorithm which does not use the fingerprint database, the performance of RMoS is superior to that of the other three floor algorithms under all tested scenarios in both buildings. The RMoS floor algorithm can achieve $100 \%$ correct floor determination for all three scenarios tested in Building A (the green lines in Figure 9(a)). However, for the 8-RN-failure scenario in Building $\mathrm{B}$, the performance of the RMoS floor algorithm is slightly lower (the solid green line in Figure 9(b)), although its percentage of correct floor determination at $95.1 \%$ is still higher than that for those of the other floor algorithms. The reason that the RMoS floor algorithm is robust to the RN failure is that its floor determination process utilizes the online RSSs that the target node receives from the working RNs in the building. Consequently, the faulty RNs have very little to no effect on the RMoS floor computing procedures. However, the performance of the RMoS algorithm is slightly lower under the 8-RN-failure scenario in the bigger building. This is because the coverage of the wireless signal is not sufficient to cover a building which is large (i.e., only $4 \mathrm{RNs}$ per $5,625 \mathrm{~m}^{2}$ ). This causes the RSSs at the target node from the RN to be very weak or to be unable to receive at all. Moreover, if the localization system is under the failure state (e.g., 8RN failure), these RSSs will cause a shift of the confidence interval for the RSS summations on each floor. This will cause the RMoS algorithm to identify the wrong floor. In other words, we can conclude that, under the state where there are nonoperating RNs, the quality and coverage of RSSs will have impacts on the performance of the RMoS technique. One of the solutions for avoiding this situation is to design a localization system such that the number and the location of the RNs should be optimized and provisioned to support the robust operation both during a normal situation and when there is a failure of some RNs. This is the next step in our future research work.

\section{Conclusion}

In this paper, we present the robust floor determination algorithm for the indoor multistory positioning systems. The proposed algorithm is called the RMoS floor algorithm. Extensive experiments were conducted to compare the proposed algorithm with the other floor algorithms previously presented in the literature. Experimental results showed that the RMoS floor algorithm outperformed the other algorithms and was able to achieve the highest percentage of correct floor determination at up to $100 \%$ in both fault-free and $R N$ failure scenarios. Specifically, the RMoS floor algorithm was able to achieve greater than $95 \%$ correct floor determination under the RN-failure scenario in which $40 \%$ of RNs in the building failed. Thus, we can say that our proposed RMoS floor algorithm is fault tolerant and robust to the $R N$-failure scenarios considered in our studies.

Our future research work will investigate how to optimize the indoor multifloor positioning system such that the floor 
algorithm can provide robust operation, both during the normal situation and when there is a failure of some RNs in either small or big buildings.

\section{Competing Interests}

The authors declare that there is no conflict of interests regarding the publication of this paper.

\section{Acknowledgments}

This work was supported in part by Suranaree University of Technology and the Office of the Higher Education Commission under the NRU project of Thailand and the National Research Council of Thailand (NRCT).

\section{References}

[1] H. Liu, H. Darabi, P. Banerjee, and J. Liu, "Survey of wireless indoor positioning techniques and systems," IEEE Transactions on Systems, Man and Cybernetics Part C: Applications and Reviews, vol. 37, no. 6, pp. 1067-1080, 2007.

[2] Y. Gu, A. Lo, and I. Niemegeers, "A survey of indoor positioning systems for wireless personal networks," IEEE Communications Surveys \& Tutorials, vol. 11, no. 1, pp. 13-32, 2009.

[3] K. Pahlavan and P. Krishnamurthy, Principles of Wireless Access and Localization, John Wiley \& Sons, New York, NY, USA, 2013.

[4] S. Gansemer, U. Großmann, and S. Hakobyan, "RSSI-based Euclidean distance algorithm for Indoor Positioning adapted for the use in dynamically changing WLAN environments and multi-level buildings," in Proceedings of the International Conference on Indoor Positioning and Indoor Navigation (IPIN '10), pp. 1-6, September 2010.

[5] S. Al-Ahmadi, T. A. Rahman, M. R. Kamarudin, M. H. Jamaluddin, and A. I. Omer, "Single-phase wireless LAN based multifloor indoor location determination system," in Proceedings of the IEEE 17th International Conference on Parallel and Distributed Systems (ICPADS '11), pp. 1057-1062, IEEE, Tainan, Taiwan, December 2011.

[6] N. Marques, F. Meneses, and A. Moreira, "Combining similarity functions and majority rules for multi-building, multi-floor, WiFi positioning," in Proceedings of the International Conference on Indoor Positioning and Indoor Navigation (IPIN '12), pp. 1-9, Sydney, Australia, November 2012.

[7] P. Kriz, F. Maly, and T. Kozel, "Improving indoor localization using bluetooth low energy beacons," Mobile Information Systems, vol. 2016, Article ID 2083094, 11 pages, 2016.

[8] Y. U. Lee and M. Kavehrad, "Two hybrid positioning system design techniques with lighting LEDs and ad-hoc wireless network," IEEE Transactions on Consumer Electronics, vol. 58, no. 4, pp. 1176-1184, 2012.

[9] IEEE, "IEEE standard for local and metropolitan area networks-part 15.4: Low-Rate Wireless Personal Area Networks (LR-WPANs)," IEEE Standard 802.15.4e-2012, 2011.

[10] D. Dardari, P. Closas, and P. M. Djurić, "Indoor tracking: theory, methods, and technologies," IEEE Transactions on Vehicular Technology, vol. 64, no. 4, pp. 1263-1278, 2015.

[11] Z. Farid, R. Nordin, M. Ismail, and N. F. Abdullah, "Hybrid indoor-based WLAN-WSN localization scheme for improving accuracy based on artificial neural network," Mobile Information Systems, vol. 2016, Article ID 6923931, 11 pages, 2016.
[12] S. Lee, I. Lim, and J. Lee, "Method for improving indoor positioning accuracy using extended kalman filter," Mobile Information Systems, vol. 2016, Article ID 2369103, 15 pages, 2016.

[13] M. Bal, H. Xuet, W. Shen, and H. Ghenniwa, "A 3-D indoor location tracking and visualization system based on wireless sensor networks," in Proceedings of the IEEE International Conference on Systems Man and Cybernetics (SMC '10), pp. 15841590, IEEE, Istanbul, Turkey, October 2010.

[14] F. Alsehly, T. Arslan, and Z. Sevak, "Indoor positioning with floor determination in multi story buildings," in Proceedings of the International Conference on Indoor Positioning and Indoor Navigation (IPIN '11), pp. 1-7, Guimarães, Portugal, September 2011.

[15] H.-H. Liu and Y.-N. Yang, "WiFi-based indoor positioning for multi-floor environment," in Proceedings of the IEEE Region 10 Conference: Trends and Development in Converging Technology Towards 2020 (TENCON '11), pp. 597-601, November 2011.

[16] P. Gupta, S. Bharadwaj, S. Ramakrishnan, and J. Balakrishnan, "Robust floor determination for indoor positioning," in Proceedings of the 20th National Conference on Communications (NCC '14), pp. 1-6, March 2014.

[17] Y.-C. Lee and S. H. Park, "Localization method for mobile robots moving on stairs in multi-floor environments," in Proceedings of the IEEE International Conference on Systems, Man and Cybernetics (SMC '14), pp. 4014-4020, IEEE, San Diego, Calif, USA, October 2014.

[18] K. Maneerat and C. Prommak, "An enhanced floor estimation algorithm for indoor wireless localization systems using confidence interval approach," International Journal of Computer, Electrical, Automation, Control and Information Engineering, vol. 8, no. 7, pp. 1182-1186, 2014.

[19] S. He and S. G. Chan, "Wi-Fi fingerprint-based indoor positioning: recent advances and comparisons," IEEE Communications Surveys \& Tutorials, vol. 18, no. 1, pp. 466-490, 2016.

[20] C. Khauphung, P. Keeratiwintakorn, and K. Kaemarungsi, “On robustness of centralized-based location determination using WSN," in Proceedings of the 14th Asia-Pacific Conference on Communications (APCC '08), pp. 1-5, October 2008.

[21] W.-C. Lai, Y.-Y. Su, C.-M. Lee et al., "A survey of secure fingerprinting localization in wireless local area networks," in Proceedings of the 12th International Conference on Machine Learning and Cybernetics (ICMLC '13), pp. 1413-1417, IEEE, Tianjin, China, July 2013.

[22] B. Mager, P. Lundrigan, and N. Patwari, "Fingerprint-based device-free localization performance in changing environments," IEEE Journal on Selected Areas in Communications, vol. 33, no. 11, pp. 2429-2438, 2015.

[23] Y. Tian, B. Denby, I. Ahriz, P. Roussel, and G. Dreyfus, "Robust indoor localization and tracking using GSM fingerprints," Eurasip Journal on Wireless Communications and Networking, vol. 2015, no. 1, article 157, 2015.

[24] T. Sathyan and M. Hedley, "Fast and accurate cooperative tracking in wireless networks," IEEE Transactions on Mobile Computing, vol. 12, no. 9, pp. 1801-1813, 2013.

[25] C. Laoudias, M. P. Michaelides, and C. G. Panayiotou, "Fault detection and mitigation in WLAN RSS fingerprint-based positioning," in Proceedings of the International Conference on Indoor Positioning and Indoor Navigation (IPIN '11), pp. 1-7, Guimaraes, Guimarães, September 2011.

[26] R. K. Jain, The Art of Computer Systems Performance Analysis: Techniques for Experimental Design, Measurement, Simulation, and Modeling, Wiley- Interscience, New York, NY, USA, 1991. 

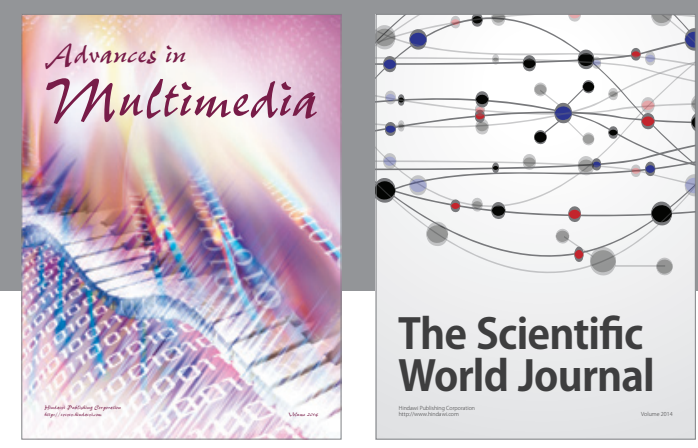

The Scientific World Journal
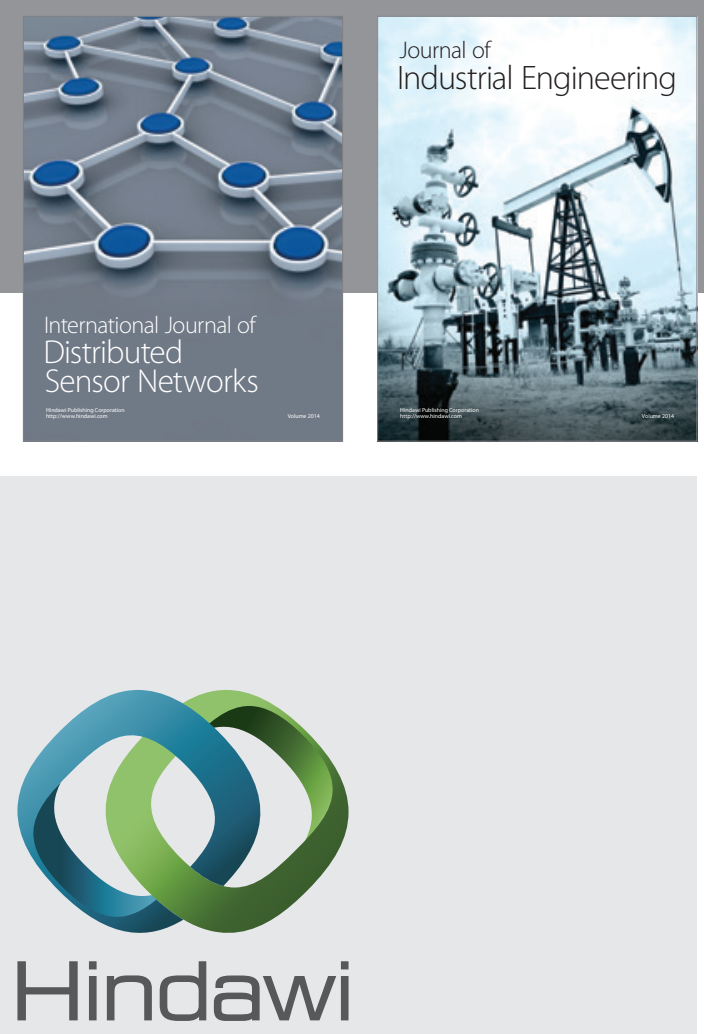

Submit your manuscripts at

http://www.hindawi.com

\section{Computer Networks} and Communications
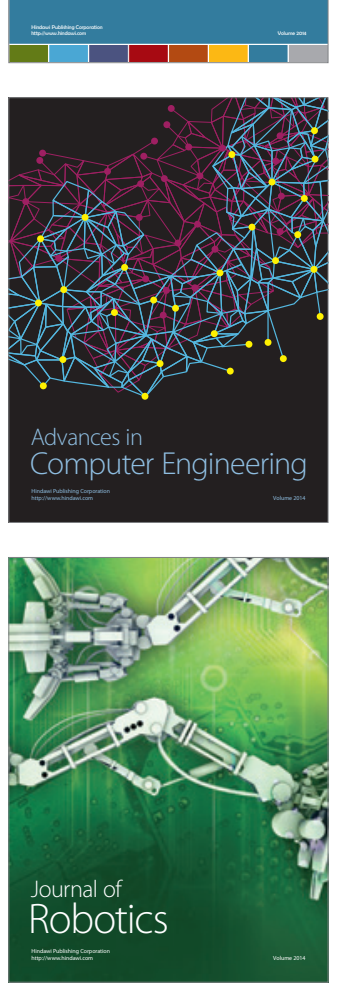
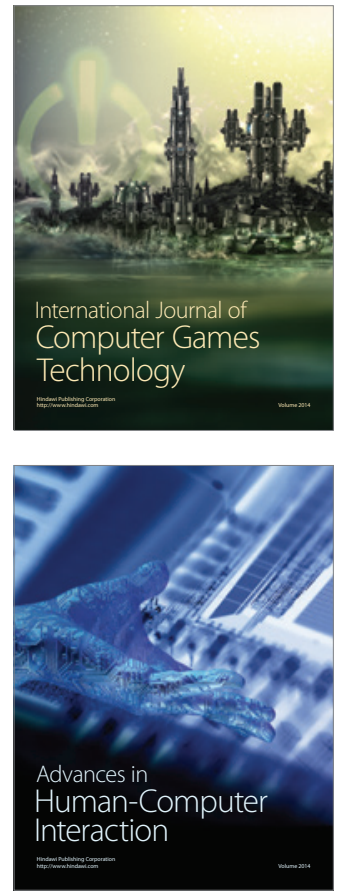
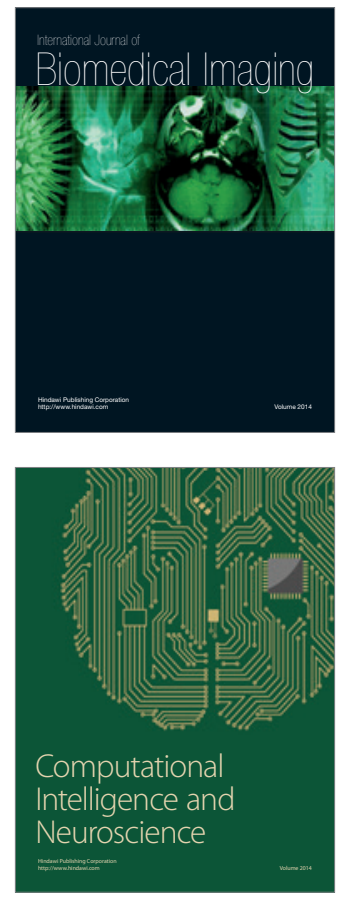
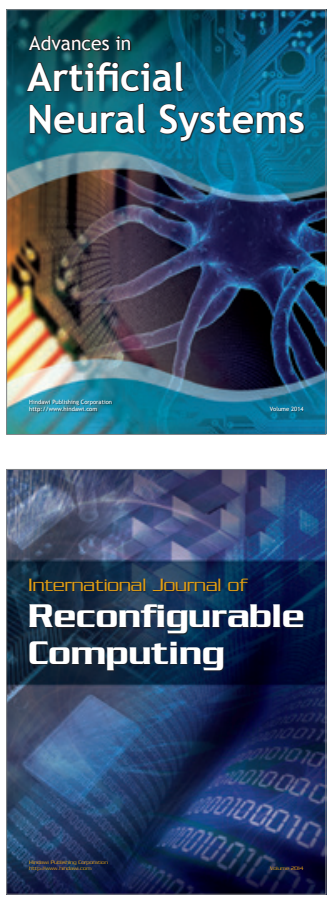
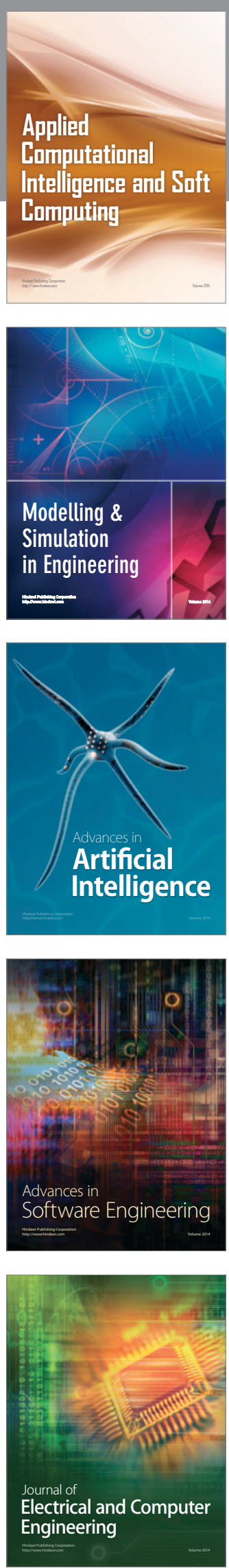